\title{
Quantum Spin Dimers from Chiral Dissipation in Cold-Atom Chains
}

\author{
Tomás Ramos, ${ }^{1,2, *}$ Hannes Pichler, ${ }^{1,2}$ Andrew J. Daley, ${ }^{3,4}$ and Peter Zoller ${ }^{1,2}$ \\ ${ }^{1}$ Institute for Quantum Optics and Quantum Information of the Austrian Academy of Sciences, 6020 Innsbruck, Austria \\ ${ }^{2}$ Institute for Theoretical Physics, University of Innsbruck, 6020 Innsbruck, Austria \\ ${ }^{3}$ Department of Physics and SUPA, University of Strathclyde, Glasgow G4 0NG, United Kingdom \\ ${ }^{4}$ Department of Physics and Astronomy, University of Pittsburgh, Pittsburgh, Pennsylvania 15260, USA
}

\begin{abstract}
We consider the nonequilibrium dynamics of a driven dissipative spin chain with chiral coupling to a one-dimensional (1D) bosonic bath, and its atomic implementation with a two-species mixture of cold quantum gases. The reservoir is represented by a spin-orbit coupled 1D quasicondensate of atoms in a magnetized phase, while the spins are identified with motional states of a separate species of atoms in an optical lattice. The chirality of reservoir excitations allows the spins to couple differently to left- and right-moving modes, which in our atomic setup can be tuned from bidirectional to purely unidirectional. Remarkably, this leads to a pure steady state in which pairs of neighboring spins form dimers that decouple from the remainder of the chain. Our results also apply to current experiments with two-level emitters coupled to photonic waveguides.
\end{abstract}

PACS numbers: 03.65.Yz, 67.85.Jk, 42.50.Dv, 03.67.Bg

In an open quantum many-body system, the competition of particle interactions, external driving and the dissipative coupling to a quantum reservoir can result in novel scenarios for the formation of strongly correlated quantum states [1]. This is not only of interest as a nonequilibrium condensed matter problem per se [2-9], but dissipatively prepared entangled states also provide a potential resource for quantum information tasks [1015]. Quantum optical systems of cold atoms or solid-state impurities provide a natural setting for such open manybody quantum systems. The paradigmatic example is given by an ensemble of two-level atoms driven by laser light, and coupled to a photonic reservoir [16-19], e.g., as one-dimensional (1D) engineered photonic band gap materials [20]. These model systems can be described as a collection of spin- $1 / 2$ systems, which via the photonic modes interact with long-range dipole-dipole interactions, and exhibit collective and enhanced decay into radiation modes of photonic structures. The realization of such Dicke-type models [21, 22] coupled to low-dimensional quantum reservoirs, and the observation of the associated dynamical quantum phases and phase transitions are, at present, an outstanding challenge in quantum optics [23-26].

In the present work, we introduce a realization of dissipative quantum magnetism based on cold atoms in optical lattices [27, 28], where the quantum reservoir is represented by phononic degrees of freedom of a 1D spin-orbit coupled Bose-Einstein quasicondensate (quasi-BEC) [2935]. This model system provides a faithful and experimentally realistic representation of a chain of driven spin-1/2 particles coupled to a $1 \mathrm{D}$ bosonic bath. Crucially, spin-orbit coupling (SOC) makes the reservoir chiral, with the spins coupling differently to the left and right propagating modes, $\gamma_{L} \neq \gamma_{R}$ [cf. Fig. 1(a)]. This asymmetry is, moreover, tunable via the atomic parameters, making it possible to engineer the spin-bath cou- pling from purely unidirectional to fully bidirectional.

To describe the dynamics of our 1D spin chain, we derive a quantum optical master equation for the reduced system density matrix $\rho(t)$, tracing over the reservoir degrees of freedom. This equation contains both longrange dipolar spin interactions, as mediated by the exchange of Bogoliubov excitations, and collective dissipative terms. Remarkably, at long times the system evolves to a pure many-body state of quantum spin dimers,

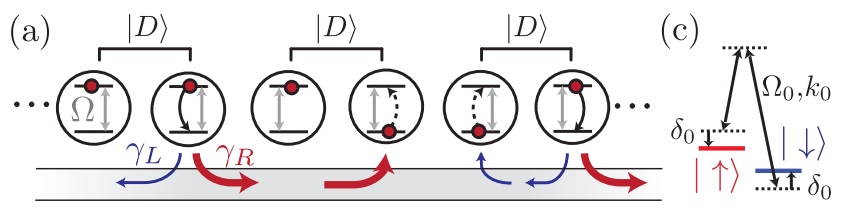

(b)

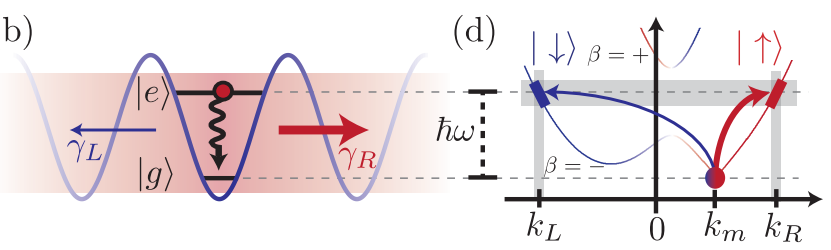

Figure 1. The 1D spin chain coupled to a 1D chiral bosonic reservoir. (a) Driven spins decay into right- and left-moving reservoir modes with rates $\gamma_{R}$ and $\gamma_{L}$. For $\gamma_{R} \neq \gamma_{L}$, quantum spin dimers (indicated by $|D\rangle$ ) are formed as the unique pure steady state. (b-d) Implementation with a two-species mixture of cold atoms. (b) Spins are represented by the two lowest vibrational states of atoms $a$ on each site of a 1D optical lattice, which can "decay" due to collisions with a 1D SOC quasi-BEC, representing the bath. (c) SOC of atoms $b$ due to coupling of two internal states $|\uparrow\rangle$ and $|\downarrow\rangle$ via a Raman process [29]. (d) Dispersion relations $\hbar \omega_{k \beta}$ of the bath excitations in the plane wave phase. The red and blue arrows indicate excitations of atoms $b$ from the quasi-BEC (circle at $k_{m}$ ) to wave vectors $k_{L}$ and $k_{R}$, resonant with $\hbar \omega$. 


$$
\begin{array}{r}
\rho(t) \stackrel{t \rightarrow \infty}{\longrightarrow}|\Psi\rangle\langle\Psi| \text { with }|\Psi\rangle=\bigotimes_{j=1}^{N / 2}|D\rangle_{2 j-1,2 j} . \text { Here } \\
|D\rangle_{j, l} \equiv \frac{1}{\sqrt{1+|\alpha|^{2}}}\left[|g\rangle_{j}|g\rangle_{l}+\frac{\alpha}{\sqrt{2}}\left(|g\rangle_{j}|e\rangle_{l}-|e\rangle_{j}|g\rangle_{l}\right)\right]
\end{array}
$$

is the spin-dimer state of a pair of spin- $1 / 2$ particles at lattice sites $j, l$ with $|g\rangle,|e\rangle$ denoting the corresponding ground and excited states, and $\alpha$ a parameter defined below. This result is valid for a generic range of parameters in the case of reservoirs with broken left-right symmetry and an even number of spins [cf. Fig. 1(a)]. Further, it is also of immediate relevance in the context of recent proposals and experiments for two-level systems (TLSs) coupled to a photonic chiral reservoir [36-39].

Model. - We realize a driven dissipative spin chain coupled to a 1D bosonic reservoir with a two-species mixture of quantum gases. The corresponding setup is shown in Figs. 1(b)-1(d). The spin chain is represented by spinless atoms of a first species $a$ (with mass $m_{a}$ ), trapped in a species-selective 1D optical lattice [3] of period $d$ [cf. Fig. 1(b)]. We assume filling with one atom per site and a deep lattice to completely suppress the tunneling (Mott insulator). Thus, the ground and first vibrational states of the atom at lattice site $j$ with position $x_{j}$ represent a TLS, $|g\rangle_{j}$ and $|e\rangle_{j}$, or effective spin-1/2. Other vibrational states are decoupled due to the lattice anharmonicity. We can drive these TLSs near their transition frequency $\omega$ via a Raman process with frequency $\nu$ and Rabi frequencies $\Omega_{j}$. In the rotating wave approximation (RWA), the Hamiltonian for the driven spin chain with $N$ atoms reads $\left(\sigma_{j} \equiv|g\rangle_{j}\langle e|\right)$

$$
H_{\mathrm{sys}}=\hbar \omega \sum_{j=1}^{N} \sigma_{j}^{\dagger} \sigma_{j}+\hbar \sum_{j=1}^{N}\left(\Omega_{j} \sigma_{j} e^{i \nu t}+\text { H.c. }\right) \text {. }
$$

The 1D bosonic quantum reservoir is realized with a second atomic species $b$ (with mass $m_{b}$ ). We assume, again, trapping in a 1D geometry (aligned with the optical lattice), however, with the atoms $b$ now moving freely along a homogeneous 1D wire. In addition, we prepare them in the quasi-BEC regime [34, 35, 40-42]; i.e., the linear density $\bar{\rho}$ satisfies $\hbar^{2} \bar{\rho}^{2} / m_{b} \gg k_{B} T$, $\mu$, with $T$ the temperature and $\mu$ the chemical potential. Atoms $a$ will couple to the reservoir atoms $b$ via collisional interactions. In particular, there will be resonant processes, where an atom a "decays" from $|e\rangle$ to $|g\rangle$, creating an excitation of energy $\hbar \omega$ in the reservoir gas [27, 43, 44] [cf. Fig. 1(b)]. These excitations will propagate along the wire and represent the right- and left-moving bosonic excitations constituting our 1D bath. First experiments along these lines have been realized with a three-dimensional BEC as the reservoir $[3,4]$.

A chiral reservoir with asymmetric decay of spins to left- and right-moving modes $\left(\gamma_{L} \neq \gamma_{R}\right)$ is obtained by adding SOC to the 1D quasi-BEC. Following Ref. [29], SOC with equal Rashba and Dresselhaus contributions can be implemented by coupling two internal states $|\uparrow\rangle$ and $|\downarrow\rangle$ of the reservoir atoms $b$ via Raman lasers with momentum transfer $2 \hbar k_{0}$, coupling strength $\Omega_{0}$, detuning $2 \delta_{0}$ and recoil energy $E_{0} \equiv \hbar^{2} k_{0}^{2} /\left(2 m_{b}\right)$ [cf. Fig. 1(c)]. Using an extension of Bogoliubov theory to quasicondensates [40, 42], one can diagonalize the reservoir Hamiltonian in terms of Bogoliubov-like excitations as $H_{\text {res }}=$ $\sum_{k, \beta} \hbar \omega_{k \beta} b_{k \beta}^{\dagger} b_{k \beta}$. We refer to Ref. [45] for details. Here $b_{k \beta}$ are bosonic annihilation operators for excitations with wave vector $k$ in the branch $\beta= \pm$, and $\omega_{k \beta}$ is the corresponding excitation spectrum shown in Fig. 1(d) for $\hbar \Omega_{0} \ll E_{0}$. What is crucial for our proposal is that at energies $\sim E_{0}$, there is an energy window $\sim \Omega_{0}$ in which excitations are chiral; i.e., all excitations with positive group velocity are strongly polarized along $|\uparrow\rangle$, while the ones with negative group velocity are strongly polarized along $|\downarrow\rangle$. This locking of the propagation direction to the spin is reminiscent of chiral edge modes in systems with artificial gauge fields $[56,57]$. To be specific, the excitation spectrum of Fig. 1(d) is obtained when the SOC quasi-BEC is prepared in the so-called plane wave phase [58-60] with quasicondensation at a positive wave vector $k_{m}$. This can be achieved by using a finite detuning $\delta_{0}<0$, satisfying $\bar{\rho}\left(g_{\uparrow \uparrow}-g_{\uparrow \downarrow}\right) / 2<\hbar\left|\delta_{0}\right| \ll E_{0}$, where $g_{\uparrow \uparrow}, g_{\downarrow \downarrow}, g_{\uparrow \downarrow} \geq 0$ are the 1D collisional interaction parameters of the reservoir gas. An important characteristic of this phase is that the atoms in the quasi-BEC are spin polarized, as manifested by $\bar{\rho}_{\downarrow} / \bar{\rho}_{\uparrow}<1$, where $\bar{\rho}_{\uparrow}$ and $\bar{\rho}_{\downarrow}$ are the mean densities of the different quasi-BEC spin components $\left(\bar{\rho}=\bar{\rho}_{\uparrow}+\bar{\rho}_{\downarrow}\right)$. A feature of the synthetic SOC is the tunability of this spin polarization with $\Omega_{0}$ [cf. Fig. 2(a)].

We take a quantum optical point of view in describing the system-bath interaction, which is motivated by the analogy with TLSs coupled to a 1D photonic bath in the weak coupling limit. Microscopically, it is given
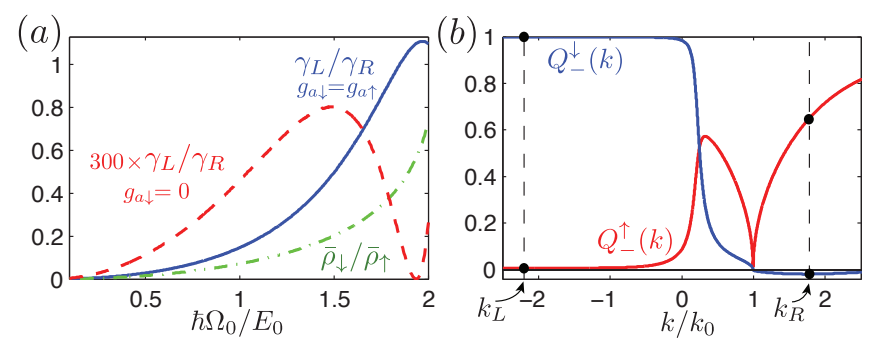

Figure 2. Tunability of decay asymmetry into chiral left- and right-moving modes (a) $\gamma_{L} / \gamma_{R}$ as a function of $\hbar \Omega_{0} / E_{0}$ for $g_{a \downarrow}=g_{a \uparrow}$ (solid line) and $g_{a \downarrow}=0$ (dashed line). The dashdotted line shows the reservoir spin polarization $\bar{\rho}_{\downarrow} / \bar{\rho}_{\uparrow}$. (b) Density fluctuation coefficients $Q_{-}^{\lambda}(k)(\lambda=\uparrow, \downarrow)$ in the lower branch for $\hbar \Omega_{0}=0.2 E_{0}$. The wave vectors for left- and rightmoving excitations $k_{s}(s=L, R)$ are indicated, where $Q_{-}^{\lambda}\left(k_{s}\right)$ show their strong spin polarization. Other parameters are $\bar{\rho}=6.14 k_{0}, m_{a} / m_{b}=2, g_{\uparrow \uparrow}=g_{\uparrow \downarrow}=g_{\downarrow \downarrow}=0.23 E_{0} / k_{0}, g_{a \uparrow}=$ $-0.37 E_{0} / k_{0}, \hbar \omega=1.46 E_{0}$ and $\delta_{0}=-0.004 E_{0}$. 
in our setup by collisional interactions between $a$ and $b$ atoms. For spinless atoms $a$, these collisions are spin conserving and reduce to interspecies density-density interactions. Therefore, density fluctuations of the reservoir atoms in a frequency band around $\omega$ provide an energy-conserving mechanism for spin decay. In terms of elementary excitations, they can be written as $\delta \rho_{\lambda}=$ $\sqrt{\bar{\rho}_{\lambda} / L} \sum_{k, \beta= \pm} Q_{\beta}^{\lambda}(k) b_{k \beta} e^{i\left(k-k_{m}\right) x}+$ H.c. [42, 45], where $L$ is a quantization length and the coefficients $Q_{\beta}^{\lambda}(k)$ (with $\lambda \in\{\uparrow, \downarrow\}$ ) reflect the spin-polarization of excitations [cf. Fig. 2(b)]. By placing the TLS transition frequency $\omega$ in the aforementioned energy window around $E_{0}$, the RWA restricts the reservoir to chiral excitations only, provided $\gamma_{L}, \gamma_{R} \ll \Omega_{0}, \omega$. Furthermore, we can linearize the dispersion in intervals $I_{L}$ and $I_{R}$, around the corresponding resonant wave vectors $k_{L}$ and $k_{R}$, with group velocities $v_{L}<0$ and $v_{R}>0$ [cf. Fig. 1(d)]. As a result, the interaction Hamiltonian can be written in a form reminiscent of the prototypical quantum optical RWA Hamiltonian as (cf. Ref. [45])

$$
H_{\mathrm{int}}=i \hbar \sum_{s=L, R} \sqrt{\frac{\gamma_{s}\left|v_{s}\right|}{L}} \sum_{k \in I_{s}, j} \sigma_{j}^{\dagger} b_{k,-} e^{i\left(k-k_{m}\right) x_{j}}+\text { H.c. }
$$

with decay rates into the left and right propagating modes $(s=R, L)$ given by

$$
\gamma_{s} \equiv \frac{\eta\left(k_{s}\right) e^{-\eta\left(k_{s}\right)}}{\hbar^{2}\left|v_{s}\right|}\left(\sum_{\lambda=\uparrow, \downarrow} g_{a \lambda} \sqrt{\bar{\rho}_{\lambda}} Q_{-}^{\lambda}\left(k_{s}\right)\right)^{2}
$$

Here $g_{a \uparrow}, g_{a \downarrow}$ are the collisional couplings between $a$ and $b$ atoms, and $\eta(k) \equiv\left(E_{0} / \hbar \omega\right)\left(m_{b} / m_{a}\right)\left[\left(k-k_{m}\right) / k_{0}\right]^{2}$.

The physical origin of the decay asymmetry $\gamma_{R} \neq \gamma_{L}$ is primarily the preparation of the reservoir in the plane wave phase at $k_{m}>0$. For $\hbar \Omega_{0} \ll E_{0}$, the reservoir atoms are strongly spin polarized $\bar{\rho}_{\uparrow} \gg \bar{\rho}_{\downarrow}$ [cf. Fig. $\left.2(\mathrm{a})\right]$, suppressing the creation of left-moving excitations in the spin-conserving collisions due to the small overlap of the spin wave functions. In addition, creating left- or rightmoving excitations requires different momentum transfers [cf. Fig. 1(d)] which also give rise to an asymmetry, reflected by the coupling constants $\eta\left(k_{s}\right)$. As illustrated in Fig. 2(a), the decay asymmetry can be tuned with $\Omega_{0}$ from essentially unidirectional $\gamma_{L} / \gamma_{R} \ll 1$ to fully bidirectional $\gamma_{L} / \gamma_{R}=1$. Another mechanism for an asymmetry is provided in the case of spin-dependent collisions $\left(g_{a \uparrow} \neq g_{a \downarrow}\right)$. In particular, for $g_{a \uparrow} \gg g_{a \downarrow}$, there is predominant decay to the right-moving modes. Remarkably, there are parameters for which $Q_{-}^{\uparrow}\left(k_{L}\right)=0$ (cf. Ref. [45]), making it possible to realize an ideal cascaded spin chain with $\gamma_{L}=0$, if $g_{a \downarrow}=0$ [cf. Fig. 2(a)].

Master equation. - We derive a master equation for the reduced density operator $\rho(t)$ of the spin chain by eliminating the reservoir atoms in the Born-Markov approximation [27, 61]. For $\hbar \omega \gg k_{B} T$, and neglecting retardation effects provided $\gamma_{s} \ll 2 \pi\left|v_{s}\right| /(N d)$ [16, 62], we find

$$
\dot{\rho}=-(i / \hbar)\left[H_{\mathrm{sys}}, \rho\right]+\mathcal{L}_{\mathrm{B}} \rho+\mathcal{L}_{\mathrm{C}} \rho,
$$

where $H_{\text {sys }}$ is defined in Eq. (2) and the Liouvillian terms describing reservoir-mediated interactions read

$$
\begin{aligned}
& \mathcal{L}_{\mathrm{B}} \rho \equiv \gamma_{L} \sum_{j, l}\left[-i \sin \left(\left|\phi_{j l}\right|\right)\left[\sigma_{l}^{\dagger} \sigma_{j}, \rho\right]+\cos \left(\left|\phi_{j l}\right|\right) \mathcal{D}\left(\sigma_{j}, \sigma_{l}\right) \rho\right], \\
& \mathcal{L}_{\mathrm{C}} \rho \equiv \frac{\Delta \gamma}{2} \sum_{j} \mathcal{D}\left(\sigma_{j}, \sigma_{j}\right) \rho+\Delta \gamma \sum_{j>l}\left(e^{-i \phi_{j l}}\left[\sigma_{j}, \rho \sigma_{l}^{\dagger}\right]+\text { H.c. }\right) .
\end{aligned}
$$

In writing Eq. (5) we used the notation $\mathcal{D}(a, b) \rho \equiv$ $2 a \rho b^{\dagger}-b^{\dagger} a \rho-\rho b^{\dagger} a$ and assumed $\Delta \gamma \equiv \gamma_{R}-\gamma_{L} \geq 0$. Additionally, we defined phase factors $\phi_{j l} \equiv\left(x_{j}-x_{l}\right)\left(k_{R}-\right.$ $\left.k_{L}\right) / 2$, and redefined $\sigma_{j} \rightarrow \sigma_{j} e^{-i\left(k_{R}+k_{L}-2 k_{m}\right) x_{j} / 2}$ and $\Omega_{j} \rightarrow \Omega_{j} e^{i\left(k_{R}+k_{L}-2 k_{m}\right) x_{j} / 2}$. The Liouvillian $\mathcal{L}_{B}$ is familiar from TLSs coupled to a symmetric (bidirectional) $1 \mathrm{D}$ waveguide [16, 23]. It contains a coherent (Hamiltonian) part, describing infinite-range dipole-dipole interactions and an incoherent part with "quantum jump operators" [61] associated with infinite-range superradiant collective decay. Its strength is given by the smaller of the decay rates $\gamma_{L}$. The last term, $\mathcal{L}_{C}$, is the Liouvillian of a cascaded quantum system [10, 61], i.e., where bath excitations can only move to the right. Its strength is given by $\Delta \gamma$ and thus it appears only if the left-right symmetry is broken.

Quantum spin dimers as the steady state.- We consider a situation where the lattice spacing $d$ is commensurate with the wavelength of the reservoir excitations, $\left(k_{R}-k_{L}\right) d=4 \pi n$ ( $n$ is an integer [63]), so that the dipole-dipole interactions vanish. In addition, we assume that all spins are driven homogeneously, $\Omega_{j}=\Omega$, and onresonance, $\nu=\omega$.

We note that for $\Delta \gamma=0$, Eq. (5) reduces to a totally symmetric Dicke model, where a nonequilibrium quantum phase transition at a critical driving $\Omega_{c} \equiv N \gamma_{L} / 4$ has been predicted $[21,23]$. In this case, only coupling within the so-called Dicke manifolds is allowed, which leads to multiple steady states. In contrast, when $\Delta \gamma \neq 0$ this symmetry is broken and the steady state is unique. Remarkably, for an even number of spins, the steady state is pure and it dimerizes; i.e., each spin pairs up with one of its neighbors in the entangled state $|D\rangle$ given in Eq. (1) with the singlet fraction $\alpha=2 i \sqrt{2} \Omega^{*} / \Delta \gamma$. Such a dimerized state represents a dark state of the drivendissipative many-body dynamics [64], where excitations are exchanged between two adjacent spins, but they do not escape from the pair due to quantum interference. For the ideal cascaded case $\left(\gamma_{L}=0\right)$, Ref. [10] has previously discussed such "cooling to dimers" with engineered optomechanical systems. In Ref. [45], we give a formal proof that this dimerization is in fact the generic steady state of Eq. (5) for the whole range $0 \leq \gamma_{L} / \gamma_{R}<1$.

To gain insight into how a spin chain dynamically purifies and arranges itself into dimers, we numerically 

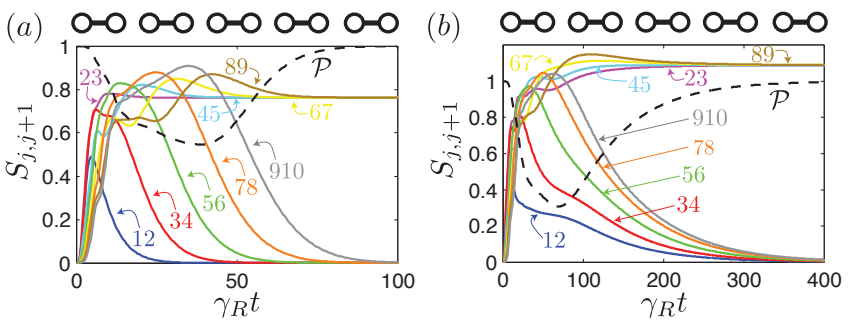

(c) $\mathrm{O}-\mathrm{O}$ O- $\mathrm{O}$ O- $\mathrm{O}$ O- $\mathrm{O} \cdots$
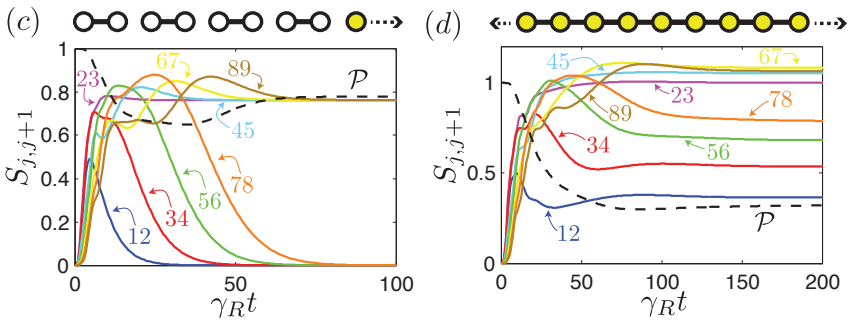

Figure 3. Dynamical formation of spin dimers as the unique steady state of the driven-dissipative spin chain. We plot the entropy $S_{j, j+1}(t)$ of all adjacent spin pairs (solid lines) and the purity $\mathcal{P}(t)$ of the total state (black dashed line) for the initial condition $|\Psi(0)\rangle=\bigotimes_{j=1}^{N}|g\rangle_{j}$. Results are shown for $\Omega=0.5 \gamma_{R}$ and (a) $N=10, \gamma_{L}=0$, (b) $N=10, \gamma_{L}=0.4 \gamma_{R}$, (c) $N=9, \gamma_{L}=0$, (d) $N=9, \gamma_{L}=0.4 \gamma_{R}$.

calculate the time evolution of the purity of the total state $\mathcal{P} \equiv \operatorname{Tr}\left\{\rho^{2}\right\}$, and the entropy of adjacent spin pairs $S_{j, j+1} \equiv-\operatorname{Tr}\left\{\rho_{j, j+1} \ln \left(\rho_{j, j+1}\right)\right\}$. Here $\rho_{j, l}$ is the reduced density operator for spins $j$ and $l$. The formation of pure dimers is manifested by $\mathcal{P}(t) \rightarrow 1$ and $S_{2 j-1,2 j}(t) \rightarrow 0$, $\forall j=1, \ldots, N / 2$, as shown in Figs. 3(a) and 3(b). For any ratio $\gamma_{L} / \gamma_{R}<1$, pairs are purified "from left to right," but only in the cascaded limit does this happen successively at a constant speed [cf. Fig. 3(a)]. The time scale $t_{\mathrm{ss}}$ to reach the steady state increases with $\gamma_{L} / \gamma_{R}$. In the limit $\gamma_{L} / \gamma_{R} \rightarrow 1$, we numerically find the scaling $t_{\mathrm{ss}} \sim\left(1-\gamma_{L} / \gamma_{R}\right)^{-4}$ for small system sizes (cf. Ref.[45]). When the number of spins is odd, it is not possible for all of them to pair up in dimers. Nevertheless, in the cascaded limit dimers are still formed, leaving only the last unpaired spin in a mixed state [cf. Fig. 3(c)]. The excitations emitted by this last spin propagate only to the right and do not affect the dimers on its left. On the other hand, if excitations can also propagate to the left, no dimers are formed because the output of an unpaired spin breaks them up [cf. Fig. 3(d)].

To ensure robustness of dissipative dimerization, we studied numerically the effect of various imperfections on the steady state of Eq. (5), reflected by the pair purities $\mathcal{P}_{2 j-1,2 j} \equiv \operatorname{Tr}\left\{\left(\rho_{2 j-1,2 j}\right)^{2}\right\}$. In general, imperfections give rise to an incomplete decoupling of spin pairs from the rest of the chain, as Fig. 4(a) illustrates for deviations from the commensurability condition, quantified by $\epsilon \equiv\left(k_{R}-k_{L}\right) d-4 \pi n$. We observe particular robustness for low $\gamma_{L} / \gamma_{R}$ and a decrease in the pair-purities from left to right. However, already for $\epsilon \lesssim 0.1$ we ob-
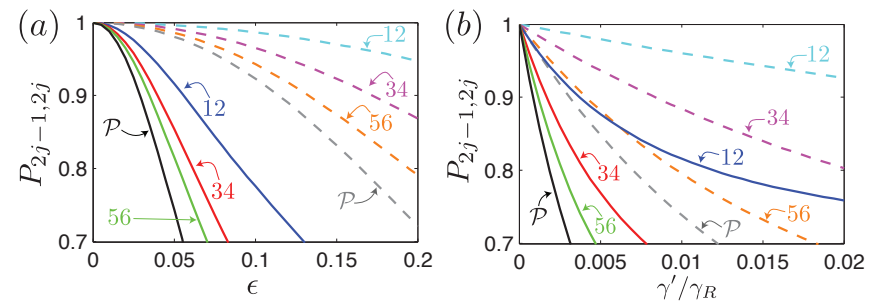

Figure 4. Robustness of the dimerized steady state against imperfections for $N=6$. (a) Pair purities $\mathcal{P}_{2 j-1,2 j}$ and total purity $\mathcal{P}$ as a function of $\epsilon$ (see text), for $\gamma_{L}=0.1$ (dashed line) and $\gamma_{L}=0.4 \gamma_{R}$ (solid line). (b) $\mathcal{P}_{2 j-1,2 j}$ and $\mathcal{P}$ as a function of decay outside the 1D bath $\gamma^{\prime}$, for $\gamma_{L}=0$ (dashed line) and $\gamma_{L}=0.4 \gamma_{R}$ (solid line). We fix $\Omega=0.5 \gamma_{R}$.

tain $\mathcal{P}_{2 j-1,2 j} \gtrsim 0.9$, when $\gamma_{L}=0.1 \gamma_{R}$. Qualitatively, the same behavior is observed for deviations in the detuning and phases of the coherent driving field. On the other hand, on-site decay outside the 1D reservoir leads to a significant decrease of the purities [cf. Fig.4(b)]. This could be a concern for implementations with photonic waveguides $[17,20]$. However, in the setup proposed here, such processes are only weakly induced (e.g., by classical noise [65]), and thus they expected to be negligible compared with $\gamma_{R}$.

Estimates. - We consider a quasi-BEC of ${ }^{87} \mathrm{Rb}$ with $E_{0} /(2 \pi \hbar) \approx 3.5 \mathrm{kHz}, 2\left|\delta_{0}\right| \gtrsim 25 \mathrm{~Hz}[29], T=5 n K$, $\bar{\rho}=48 \mu \mathrm{m}^{-1}$ (e.g. with 4800 atoms confined to $L \sim$ $100 \mu \mathrm{m}[34,35,66])$, and a transverse trapping frequency $\omega_{\perp} /(2 \pi)=10 \mathrm{kHz}$. For the spin chain we consider $\mathrm{Yb}$, because it is spinless and heavy $\left(m_{a} / m_{b} \approx 2\right)$ (cf. Ref. [45]). With interspecies scattering lengths between ${ }^{87} \mathrm{Rb}$ and ${ }^{172} \mathrm{Yb}$ of $a_{a \uparrow}=a_{a \downarrow} \approx-160.7 a_{\mathrm{Bohr}}$ [67] and $\hbar \omega /(2 \pi) \approx$ $5.3 \mathrm{kHz}$, one obtains decay rates $\gamma_{R} /(2 \pi) \sim 100 \mathrm{~Hz}$, with asymmetries $10^{-3}<\gamma_{L} / \gamma_{R}<1.1$ [cf. Fig. 2(a)]. These rates validate a posteriori the RWA and Markov approximations, as well as neglecting retardation effects for systems up to $N \sim 30$ spins spaced by $d \sim 800 \mathrm{~nm}$. On the other hand, these "quantum optical" approximations can also be deliberately violated in our setup to study retardation and non-Markovian effects outside the validity of the master equation treatment. We note that heating due to photon scattering [33] in the ${ }^{87} \mathrm{Rb}$ SOC quasi$\mathrm{BEC}$ is negligible on time scales related to the formation of dimers (cf. [45, 68]).

Outlook. - We have shown how SOC in an atomic gas can be used to engineer a chiral reservoir for spin chains. The tunable asymmetry of the coupling to leftand right-moving excitations leads to a pure steady state in which neighboring spins are dimerized, representing a novel form of dissipative quantum magnetism [69, 70]. While the cold-atom realization provides particular advantages, our results also apply to implementations with photons [37-39]. We have shown [71] that the present results generalize to the dissipative formation of pure many-body states of spin- $1 / 2$ tetramers, hexamers, etc., 
by appropriate driving patterns [10]. This multipartite entanglement can be detected via the Fisher information [72], which recently has been measured in cold-atom experiments [73].

We thank M. A. Baranov, Y. Castin, N. Goldman, A. González-Tudela, G. I. Martone, C. Mora, J. V. Porto, S. L. Rolston and Y. Li for helpful discussions. Work in Innsbruck was supported by the ERC Synergy Grant UQUAM, the EU grant SIQS and the Austrian Science Fund through SFB FOQUS. T. R. further acknowledges financial support from BECAS CHILE.

* tomas.ramos@uibk.ac.at

[1] For a review see M. Müller, S. Diehl, G. Pupillo, and P. Zoller, Adv. At. Mol. Opt. Phys. 61, 1 (2012).

[2] S. Diehl, A. Micheli, A. Kantian, B. Kraus, H. P. Büchler, and P. Zoller, Nature Phys. 4, 878 (2008).

[3] R. Scelle, T. Rentrop, A. Trautmann, T. Schuster and M. K. Oberthaler, Phys. Rev. Lett. 111, 070401 (2013).

[4] D. Chen, C. Meldgin, and B. DeMarco, Phys. Rev. A 90, 013602 (2014).

[5] M. Knap, D. A. Abanin, and E. Demler, Phys. Rev. Lett. 111, 265302 (2013).

[6] T. Prosen, and M. Znidaric, Phys. Rev. Lett. 105, 060603 (2010).

[7] D. D. Bhaktavatsala Rao, and K. Molmer, Phys. Rev. Lett. 111, 033606 (2013).

[8] A. W. Carr, and M. Saffman, Phys. Rev. Lett. 111, 033607 (2013).

[9] M. Honing, D. Muth, D. Petrosyan, and M. Fleischhauer, Phys. Rev. A 87, 023401 (2013).

[10] K. Stannigel, P. Rabl, and P. Zoller, New J. Phys. 14, 063014 (2012).

[11] H. Krauter, C. A. Muschik, K. Jensen, W. Wasilewski, J. M. Petersen, J. I. Cirac, and E. S. Polzik, Phys. Rev. Lett. 107, 080503 (2011).

[12] H. Weimer, M. Müller, I. Lesanovsky, P. Zoller, and H. P. Büchler, Nature Phys. 6, 382 (2010).

[13] F. Verstraete, M. M. Wolf, and I. J. Cirac, Nature Phys. 5, 633 (2009).

[14] J. T. Barreiro, M. Müller, P. Schindler, D. Nigg, T. Monz, M. Chwalla, M. Hennrich, C. F. Roos, P. Zoller, and R. Blatt, Nature 470, 486 (2011).

[15] Y. Lin, J.P. Gaebler, F. Reiter, T.R. Tan, R. Bowler, A. S. Sorensen, D. Leibfried and D. J. Wineland, Nature 504, 415 (2013).

[16] D. E. Chang, L. Jiang, A. V. Gorshkov, and H. J. Kimble, New J. Phys. 14, 063003 (2012).

[17] D. Reitz, C. Sayrin, R. Mitsch, P. Schneeweiss, and A. Rauschenbeutel, Phys. Rev. Lett. 110, 243603 (2013).

[18] R. Yalla, M. Sadgrove, K. P. Nayak and K. Hakuta, Phys. Rev. Lett. 113, 143601 (2014).

[19] J. D. Thompson, T. G. Tiecke, N. P. de Leon, J. Feist, A. V. Akimov, M. Gullans, A. S. Zibrov, V. Vuletić, and M. D. Lukin, Science 340, 1202 (2013).

[20] A. Goban, C.-L. Hung, S. P. Yu, J. D. Hood, J. A. Muniz, J. H. Lee, M. J. Martin, A. C. McClung, K. S. Choi, D. E. Chang, O. Painter, and H. J. Kimble, Nat. Commun. 5, 3808 (2014).
[21] D. F. Walls, P. D. Drummond, S. S. Hassan, and H. J. Carmichael, Prog. Theor. Phys. Suppl. 64, 307 (1978).

[22] M. Gross, and S. Haroche, Phys. Rep. 93, 301 (1982).

[23] A. González-Tudela, and D. Porras, Phys. Rev. Lett. 110 080502 (2013).

[24] K. Baumann, C. Guerlin, F. Brennecke, and T. Esslinger, Nature 464, 1301 (2010).

[25] L. J. Zou, D. Marcos, S. Diehl, S. Putz, J. Schmiedmayer, J. Majer, and P. Rabl, Phys. Rev. Lett. 113, 023603 (2014).

[26] A. F. van Loo, A. Fedorov, K. Lalumiere, B. C. Sanders, A. Blais and A. Wallraff, Science 342, 1494 (2013).

[27] A. J. Daley, P. O. Fedichev, and P. Zoller, Phys. Rev. A 69, 022306 (2004).

[28] H. Schwager, J. I. Cirac, and G. Giedke, Phys. Rev. A 87, 022110 (2013).

[29] Y. J. Lin, K. Jimenez-García, and I.B. Spielman, Nature 47183 (2011).

[30] S.-C. Ji, J.-Y. Zhang, L. Zhang, Z.-D. Du, W. Zheng, Y.J. Deng, H. Zhai, S. Chen, and J.-W. Pan, Nature Phys. 10, 314 (2014).

[31] L. W. Cheuk, A. T. Sommer, Z. Hadzibabic, T. Yefsah, W. S. Bakr, and M. W. Zwierlein, Phys. Rev. Lett. 109, 095302 (2012).

[32] P. Wang, Z.-Q. Yu, Z. Fu, J. Miao, L. Huang, S. Chai, H. Zhai and J. Zhang, Phys. Rev. Lett. 109095301 (2012).

[33] N. Goldman, G. Juzeliunas, P. Ohberg, and I.B. Spielman, arXiv:1308.6533 (2013).

[34] P. Krüger, S. Hofferberth, I. E. Mazets, I. Lesanovsky, and J. Schmiedmayer, Phys. Rev. Lett. 105, 265302 (2010).

[35] T. Jacqmin, J. Armijo, T. Berrada, K. V. Kheruntsyan, and I. Bouchoule, Phys. Rev. Lett. 106, 230405 (2011).

[36] J. Petersen, J. Volz, and A. Rauschenbeutel, Science 346, 67 (2014).

[37] R. Mitsch, C. Sayrin, B. Albrecht, P. Schneeweiss, and A. Rauschenbeutel, arXiv:1406.0896 (2014).

[38] I. Söllner, S. Mahmoodian, A. Javadi, and P. Lodahl, arXiv:1406.4295 (2014).

[39] A. B. Young, A. Thijssen, D. M. Beggs, L. Kuipers, J. Rarity, and R. Oulton, arXiv:1406.0714 (2014) [Phys. Rev. Lett. (to be published)].

[40] D. S. Petrov, G. V. Shlyapnikov, and J. T. M. Walraven, Phys. Rev. Lett. 85, 3745 (2000).

[41] J.O. Andersen, U. Al Khawaja, and H. T. C. Stoof, Phys. Rev. Lett. 88, 070407 (2002).

[42] C. Mora and Y. Castin, Phys. Rev. A 67053615 (2003).

[43] A. Griessner, A. J. Daley, S. R. Clark, D. Jaksch, and P. Zoller, Phys. Rev. Lett. 97, 220403 (2006).

[44] A. Griessner, A. J. Daley, S. R. Clark, D. Jaksch and P. Zoller, New J. Phys. 9, 44 (2007).

[45] See Supplemental Material at url, which includes Refs. [46-55].

[46] V. N. Popov, Functional Integrals in Quantum Field Theory and Statistical Mechanics (D. Reidel Publishing Company, 1983).

[47] M. Olshanii, Phys. Rev. Lett. 81, 938 (1998).

[48] P. Hohenberg, Phys. Rev. 158, 383 (1967).

[49] J. Kane, and L. Kadanoff, Phys. Rev. 155, 80 (1967).

[50] Y. Castin, J. Phys. IV France 116, 89 (2004).

[51] N. K. Whitlock, and I. Bouchoule, Phys. Rev. A 68, 053609 (2003).

[52] L. P. Pitaevskii, and S. Stringari, Bose-Einstein Condenstation (Oxford University Press, 2003). 
[53] K. Stannigel, P. Rabl, A. S. Sorensen, M. D. Lukin and P. Zoller, Phys. Rev. A 84, 042341 (2011).

[54] A. J. Daley, Adv. Phys. 63, 77 (2014).

[55] R. Grimm, M. Weidemüller and Y. B. Ovchinnikov, Adv. At. Mol. Opt. Phys. 42, 95 (2000).

[56] M. Atala, M. Aidelsburger, M. Lohse, J. T. Barreiro, B. Paredes, and I. Bloch, Nature Phys. 10, 588 (2014).

[57] A. Celi, P. Massignan, J. Ruseckas, N. Goldman, I. B. Spielman, G. Juzeliunas, and M. Lewenstein, Phys. Rev. Lett. 112, 043001 (2014).

[58] Y. Li, L. P. Pitaevskii, and S. Stringari, Phys. Rev. Lett. 108, 225301 (2012).

[59] S.-C. Ji, L. Zhang, X.-T. Xu, Z. Wu, Y. Deng, S. Chen, and J.-W. Pan, arXiv:1408.1755 (2014) [Phys. Rev. Lett. (to be published)].

[60] G. I. Martone, Y. Li, L. P. Pitaevskii, and S. Stringari, Phys. Rev. A 86, 063621 (2012).

[61] C. W. Gardiner and P. Zoller, Quantum Noise, 3rd Edition (Springer-Verlag, Berlin Heidelberg, 2004).

[62] P. Milonni and P. Knight, Phys. Rev. A 10, 1096 (1974).

[63] $n$ can also be half-integer, as one can reabsorb the resulting staggered phase by $\sigma_{j} \rightarrow(-1)^{j} \sigma_{j}$ and $\Omega_{j} \rightarrow(-1)^{j} \Omega_{j}$.

[64] A. S. Parkins, P. Marte, P. Zoller, and H. J. Kimble, Phys. Rev. Lett. 71, 3095 (1993).
[65] H. Pichler, J. Schachenmayer, A. J. Daley, and P. Zoller, Phys. Rev. A 87, 033606 (2013).

[66] T. P. Meyrath, F. Schreck, J. L. Hanssen, C. S. Chuu, and M. G. Raizen, Phys. Rev. A 71, 041604 (2005).

[67] M. Borkowski, P. S. Zuchowski, R. Ciurylo, P. S. Julienne, D. Kedziera, L. Mentel, P. Tecmer, F. Münchow, C. Bruni, and A. Görlitz, Phys. Rev. A 88, 052708 (2013).

[68] The corresponding heating rate could even be reduced by changing to other atomic species such as Dy or Er [74].

[69] In the Hamiltonian context, this dimerization is reminiscent of valence bond states [70].

[70] A. Auerbach, Interacting Electrons and Quantum Magnetism, 1st Edition (Springer-Verlag, Berlin Heidelberg New York, 1994).

[71] H. Pichler, T. Ramos, A. J. Daley, and P. Zoller, arXiv:1411.2963 (2014).

[72] P. Hyllus, W. Laskowski, R. Krischek, C. Schwemmer, W. Wieczorek, H. Weinfurter, L. Pezze, and A. Smerzi, Phys. Rev. A 85, 022321 (2012).

[73] H. Strobel, W. Muessel, D. Linnemann, T. Zibold, D. B. Hume, L. Pezze, A. Smerzi, and M. K. Oberthaler, Science 345, 424 (2014).

[74] S. Nascimbene, J. Phys. B, 46, 134005 (2013). 